\title{
SMart Greenhouse BaSed on the Arduino Platform
}

\author{
Hrvoje Prskalo, Jurica Trstenjak \& Bruno Trstenjak
}
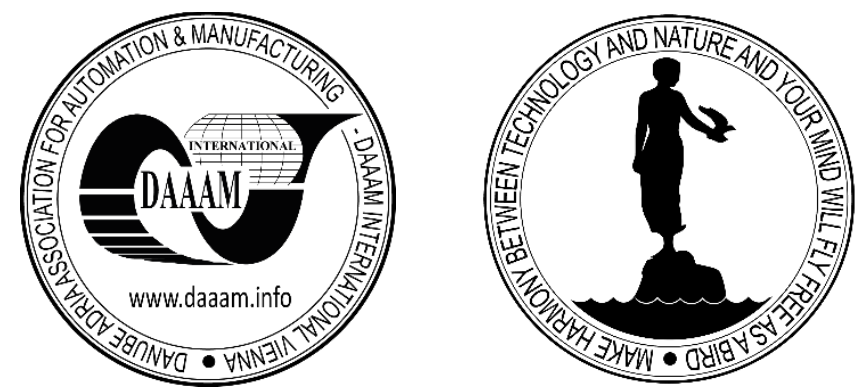

This Publication has to be referred as: Prskalo, H[rvoje]; Trstenjak, J[urica] \& Brkic, D[ragan] (2020). Smart Greenhouse Based on the Arduino Platform, Proceedings of the 31st DAAAM International Symposium, pp.0282-0289, B. Katalinic (Ed.), Published by DAAAM International, ISBN 978-3-902734-29-7, ISSN 1726-9679, Vienna, Austria DOI: $10.2507 / 31$ st.daaam.proceedings.039

\begin{abstract}
The paper describes a system for automatic control of microclimatic factors based on the Arduino platform, whose work and functionalities are shown on the model of the system itself. The Arduino Uno is a control unit of the system that, according to the measurements read from the sensors of temperature (DHT22), humidity and soil (YL69) and light intensity (photoresistor/LDR), controls the ventilation, irrigation, lighting and indication of the need for heating. To monitor the measured values from the sensor and set the optimal limits required for higher and better plant yield, a Nextion touch screen was used, with which the user can easily change the optimal limits value according to the needs of the plant culture. The paper explains the technologies used and the necessary components for system design. The connection of each component with the Arduino platform is explained in detail, accompanied by diagrams and the necessary program code for proper operation. Also, the purpose of each sensor and executive elements in terms of the automation system is explained. Finally, the created model of the system is shown with all the associated parts connected into one ensemble and placed in the appropriate places of the system model. Also, testing of the system itself was done to show the purpose and mode of operation to create optimal microclimatic conditions in the protected area. The whole system is designed to be modular, so that it can be upgraded as needed. The very cost-effectiveness of such a system is assessed by the greenhouse user himself, ie, in this case it is valid: if the payback period is less than the specified project life, the investment is acceptable. the shorter the payback period, the more justified the investment. [9]
\end{abstract}

Keywords: arduino platform; automation; Nextion display; control; sensor

\section{Introduction}

In order to achieve the highest possible yields of plants and for as many harvests per year, farmers decide to build controlled protected areas, known as greenhouses. By making a greenhouse and its automation, it is possible to control microclimatic factors such as temperature, humidity, soil moisture and light needed for the best possible growth and development of plants within the protected area. The topic of this paper is to develop and describe a system for automatic control of microclimatic conditions inside greenhouses using the development platform Arduino Uno. The platform itself has a wide range of applications today due to its low price, modularity and open source code. The final product is based on the Arduino device which controls sensors (measures temperature, humidity, light intensity and soil moisture). Depending on the measured values of the sensors, the Arduino device controls the executive elements for ventilation, irrigation, lighting and supervises the need for heating of climatic conditions in the greenhouse. As different plant cultures need different microclimatic conditions, process monitoring and control is enabled via Nextion touch screen. A model of such a system was made for the purpose of demonstration and testing. The whole system is designed to be modular, so that it can be upgraded if needed 


\section{Important}

This paper deals with the problem of poor yields of greenhouses that have no system control of important parameters, temperature and humidity. Prof. D.O.Shirsath and co-workers [10] have implemented a similar system, a greenhouse controlled by the Arduino platform, however, such a system is not very adaptable to the "ordinary" man because the parameters of the whole system change / adjust only in the program. This system uses a touch screen through which the user, as needed, enters and changes the desired parameters of the entire system. The advantage of this control is that the user does not have to have any programming knowledge. So, it can be said that such a control system is "user friendly". It could be said that the disadvantage of this system is that the soil moisture is measured in only one place (only one sensor is used), instead of the humidity being measured in several places. Of course, this can be easily solved in a real greenhouse, while this is just a scaled-down model. As a next step, instead of the classic irrigation (pipes), a device that disperses water into a water mist could. An alarm system could also be installed in the event that any of the parameters exceeded the default limits.

\section{Greenhouse plant growing and microclimate conditions}

According to [1], a greenhouse is a special type of protected environment that is completely adapted to the cultivation of plants with its shape, size and equipment. Compared to outdoor plant production, greenhouse production is possible throughout the year and shows better results and higher yields. The purpose of the greenhouse is to create the microclimatic conditions necessary for the highest possible yield and quality of plants, and by automating it, even better results are achieved.

\subsection{Greenhouse microclimate}

Optimal temperature is one of the most important factors in plant production. Temperature directly affects early ripening, quality and yield of plants. Unfavourable conditions outside the optimal limits for quality growth lead to slower plant growth and death. To avoid oscillations in day and night temperatures, the greenhouse should be equipped with a system for ventilation and space heating. According to [2], devices for the production of hot air with flue pipes are used to heat the space in the greenhouse, and their application is very wide. Also, hot water heating systems are used where the pipes are placed on the floor. Sunlight is a vegetation factor needed by plants in the process of photosynthesis, i.e. the conversion of solar energy into chemical energy of carbohydrates and other substances. [3] All crops need a certain intensity of light and a certain length of day. The intensity and quality of light depends on the geographical location, time of year, duration of sunny days.

Depending on the length of daylight, plants are divided into short-day and long-day plants. For the optimal amount of light in the greenhouse, the sun rays should fall at an angle of $90^{\circ}$. The sun rays falling at a different angle are reflected outside the greenhouse. The most important for photosynthesis are the sun rays in the wavelength range from 360 to 760 $\mu \mathrm{m}$. According to [3], assimilation lamps are used in the absence of sunlight. Furthermore, the plants need to be irrigated. There are several irrigation designs depending on the water needs of the plants and the technology of use. Irrigation performance can be divided into two groups: drip or sprinkler. In the case of drip irrigation, water pipes are placed directly on the ground below the plant, and in the case of sprinkling, the pipes are placed above the plants and spraying is done with sprayers. Humidity is important for the quality and contribution of plants, which affects the occurrence of the disease and less fertilization. Transpiration stimulates the absorption of water from the soil, and thus the absorption of important minerals. [4] Depending on the needs of the plant, the greenhouse is ventilated.

\section{Components and technologies used}

The components used in this project are listed in Table 1. The following chapters will describe the Arduino platform, the Nextion display, sensors, and other necessary electronic components. All components must be combined into one unit and program code must be written.

\subsection{Arduino platform}

The Arduino platform is an electronic platform based on the ease of use of the hardware part which is the physical electronic programmable circuit and the software part of the IDE (Integrated development environment) which is used for programming and control of the board itself. As it is open-source, it is allowed to share and rearrange it in order to create new platforms. The most important part of this platform is the microcontroller. A microcontroller is a small computer that is contained on a single integrated circuit. The Arduino platform most commonly uses an 8-bit microcontroller manufactured by ATMEL. The microcontroller, depending on the tasks it will perform, needs to be programmed. The program code for the platform itself is written in $\mathrm{C}$ and $\mathrm{C}++$ language in the free Arduino IDE software tool written in Java. The software runs on all operating systems. Communication and transfer of program code from the computer to the Arduino is done via a USB connection. [6] 


\begin{tabular}{|l|l|}
\hline Arduino Uno & Power supply 12V DC \\
\hline Nextion display & Fan \\
\hline Temperature and humidity sensor -DHT22 & Water pump \\
\hline Soil moisture sensor - YL 69 & Resistors \\
\hline Photoresistor (LDR) & MOSFET transistor \\
\hline Relay SRD-05VDC-SL-C & Plexiglass \\
\hline LED strip & \\
\hline
\end{tabular}

Table 1. Components

\subsection{Arduino Uno}

The Arduino Uno is a board that uses an ATmega328P microcontroller. The board itself has 14 digital input / output pins (6 of which can be used as a PWM output), 6 analog inputs, $16 \mathrm{MHz}$ quartz crystal, USB port, power socket, reset button and other parts shown in Figure 1. Arduino Uno also contains a UART port (Universal Asynchronous Receiver / Transmitter). The UART consists of RX and TX pins. RX is used to receive data and is located on digital pin 0 , and TX is used to send data and located on digital pin 1. Each of the 14 digital pins can be used as an output or input using the pinMode () function. When the pin is set to OUTPUT with the previous function using the digitalWrite () function, it is set to HIGH $(5 \mathrm{~V})$ or LOW $(0 \mathrm{~V})$. The pins operate at a voltage of $5 \mathrm{~V}$, and can give or receive current of $20 \mathrm{~mA}$ up to a maximum of 40mA. Table 2 shows the technical specifications of the Arduino UNO platform. [7]

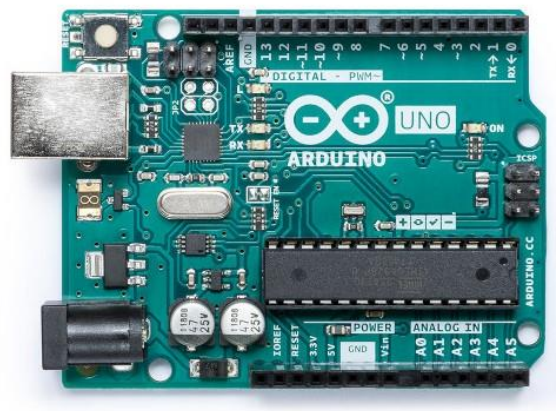

Fig. 1. Arduino Uno REV3

\begin{tabular}{|l|l|}
\hline Microcontroller & ATmega328P \\
\hline Voltage required for operation & $5 \mathrm{~V}$ \\
\hline Recommended input voltage & $7-12 \mathrm{~V}$ \\
\hline Maximum input voltage & $6-20 \mathrm{~V}$ \\
\hline Digital input / output pins & 14 (of which 6 are used for PWM output) \\
\hline Analog input pins & 6 \\
\hline Direct current for one I/O pin & $20 \mathrm{~mA}$ \\
\hline DC current for 3.3V pin & $50 \mathrm{~mA}$ \\
\hline Flash memory & $32 \mathrm{~KB}(0.5 \mathrm{~KB}$ uses bootloader) \\
\hline SRAM & $2 \mathrm{~KB}$ \\
\hline EEPROM & $1 \mathrm{~KB}$ \\
\hline Processor speed & $16 \mathrm{~Hz}$ \\
\hline
\end{tabular}

Table 2. Technical specifications of the Arduino UNO platform 


\subsection{Nextion display}

The display is HMI (Human Machine Interface). By HMI we mean a customized interface that presents processed data to the operator and through which the operator manages and monitors the process. [5] There are several types of screens, from 2.4" to 7". The model used in this project is the NX4024K032 with the dimension of $3.2^{\prime \prime}$ and the screen resolution of 400x 240 pixels. The screen is touch sensitive which will allow control of the greenhouse automation system. It uses its own FLASH memory to save the file. It needs to be connected to a 5V voltage source, grounded via a GND pin, and then the RX pin of the display should be connected to the TX pin of the Arduino and the TX pin of the display to the RX pin of the Arduino. The Arduino platform and the screen communicate via serial communication. The Nextion library must be included in the program code. To create the appearance of the screen and use its functionalities, the Nextion Editor software is used, which generates a file that is written to the FLASH memory of the screen itself via the SD card.

\subsection{Nextion editor}

Nextion Editor is development software used to build graphical user interfaces for the screen. Figure 3 shows the layout of the Nextion Editor software with the numbers indicating the different parts to create and other functionalities. Furthermore, parts of the software are described by numbers.

\subsection{DHT22}

The DHT22 or AM2302 is a capacitive relative humidity and air temperature sensor that outputs a 40-bit digital signal. It is very easy to use, but requires at least two seconds to load new values of temperature and humidity. The temperature sensor and the relative humidity sensor are connected to an 8-bit microcontroller and OTP memory through which each reading is calibrated. The DHT22 sensor has its own Single Wire protocol used for data transmission. The DHT library must be used in the program code. The sensor is very small in size and low in consumption. Compared to DHT11, this sensor is more precise, works in a larger temperature and humidity range, but is also more expensive. Figure 4 shows the DHT22 sensor and its pins are marked. The characteristics of the sensor are given in Table 3.

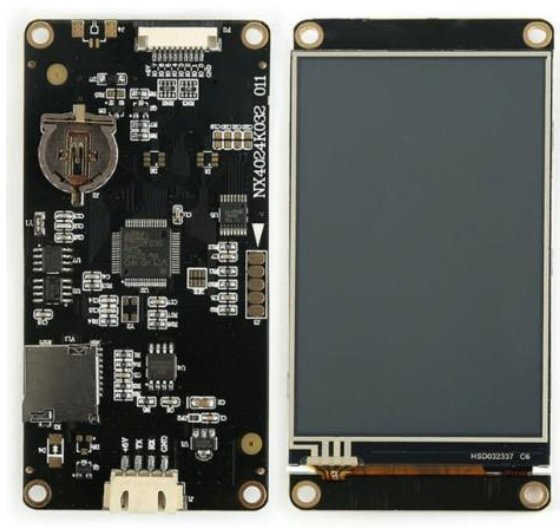

Fig. 2. Nextion screen

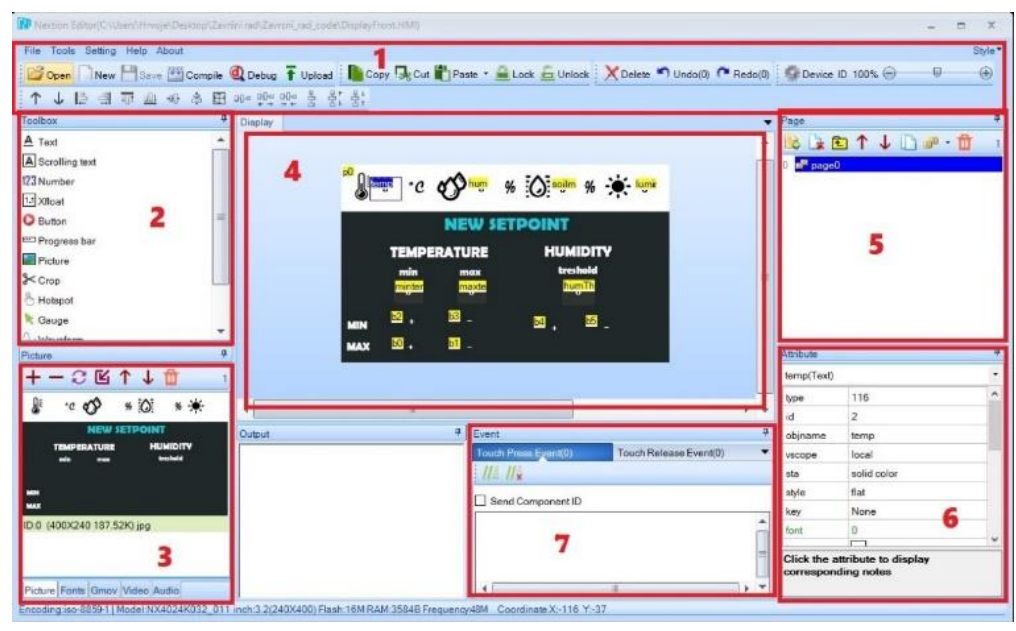

Fig. 3. Nextion editor 


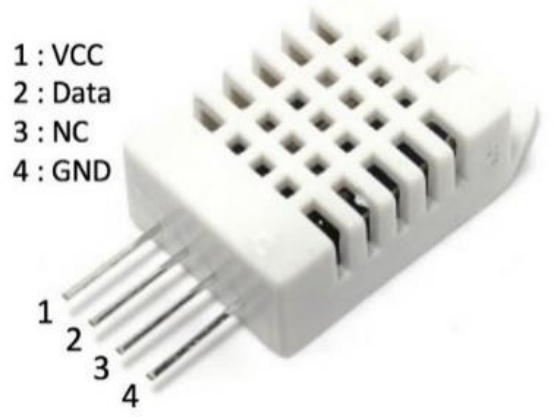

Fig. 4. DHT22 sensor

\subsection{YL69}

The type of sensor used in operation is a resistor sensor. The sensor uses two probes: two metal rods separated at a fixed distance. The rods are placed in the ground and electricity passes through them. More water in the soil means better conductivity and less resistance, and when the soil is dry the conductivity will be lower and the resistance will increase. In addition to the probes, the module also consists of a logic part to which the probes are connected. Measurement results are displayed numerically from 0-1023 and can be scaled. This sensor is very cheap, but corrosion is possible with frequent reading. Figure 5 shows the logic module and the probe

\section{System implementation}

This chapter explains how to connect each component to the Arduino platform, which is actually the control unit of the system. Relays and MOSFET transistors are used to turn the actuators on and off. The measured values from the sensor are sent by the Arduino platform to the Nextion display. The Arduino board in this case is powered via a 12V DC voltage.

\subsection{Relative humidity and temperature sensor}

The DHT22 module in this case is used to measure the internal temperature and relative humidity in the model of the greenhouse. In order for the Arduino to be able to receive and read the measured values from the sensor, it is necessary to connect the first pin on the left to $5 \mathrm{~V}$ (VCC) and the last pin to GND ground. Communication with the Arduino platform is achieved via the second pin on the left (DATA) by connecting it to the Arduino digital pin 2.

\subsection{Soil moisture sensor}

The Y1-69 sensor is used to read soil moisture. In order for the Arduino Uno to receive the measured values, the sensor must be connected according to the following instructions. The sensor consists of a probe that measures the amount of water in the soil, and a logic module. The probe is connected to the + and - pin of the logic module. A voltage of $5 \mathrm{~V}$ is connected to the VCC pin of the logic module, and GND to ground. The measurement results are obtained via the analog output A0 which is connected to the Arduino analog pin 1.

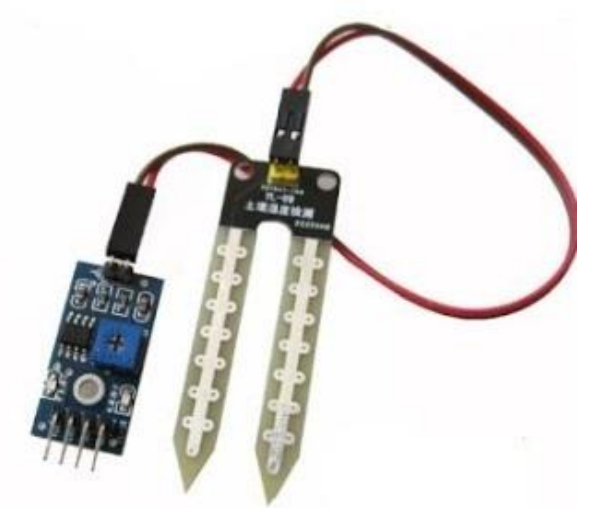

Fig. 5. YL69 sensor 


\subsection{Light intensity sensor}

A photo resistor is used to measure light intensity. In order to be able to communicate with the Arduino UNO platform, the photo resistor is connected to a $10 \mathrm{k} \Omega$ resistor and together they form a voltage divider. One pin of the photo resistor needs to be connected to a voltage of $5 \mathrm{~V}$ and the other to an analog pin 0 on the Arduino platform. The resistor is connected to the analog pin on the Arduino platform, and its other side must be grounded.

\subsection{Cooling}

Two fans cool the space in the greenhouse model when needed. Relative humidity will also decrease. In order to be able to control the fans, it is necessary to connect them to a $12 \mathrm{~V} \mathrm{DC}$ voltage via a relay. First, the + pin (red) of the relay is connected to voltage of $5 \mathrm{~V}$, and the - pin (black) must be grounded. Communication with the Arduino platform is achieved via the $\mathrm{S}$ pin of the relay module which is connected to digital pin 4 . The $12 \mathrm{~V}$ DC power supply is connected to the COM pin of the relay and the NO pin of the relay is connected to the fans (connected in parallel). Fans also need to be grounded. The connection diagram of the Arduino Uno fan and platform is shown in Figure 6.

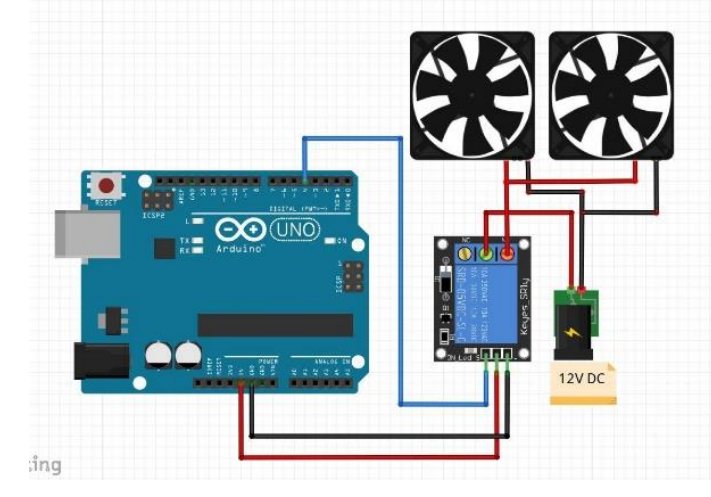

Fig. 6. Connection diagram of the sensor fan with the Arduino

\subsection{Irrigation}

The water pump is used to irrigate the soil when needed. In order to be able to control the pump, it is necessary to connect it to a $12 \mathrm{~V}$ DC voltage via a relay. First the + pin (red) of the relay is connected to a voltage of $5 \mathrm{~V}$, and the - pin (black) needs to be grounded to the Arduino GND. Communication with the Arduino platform is achieved via the $\mathrm{S}$ pin of the relay module which is connected to the digital pin 8 . The $12 \mathrm{~V}$ voltage supply is connected to the COM pin of the relay and the NO pin of the relay is connected to the pump. The pump also needs to be grounded. The connection diagram of the water pump with the Arduino is shown in Figure 7.

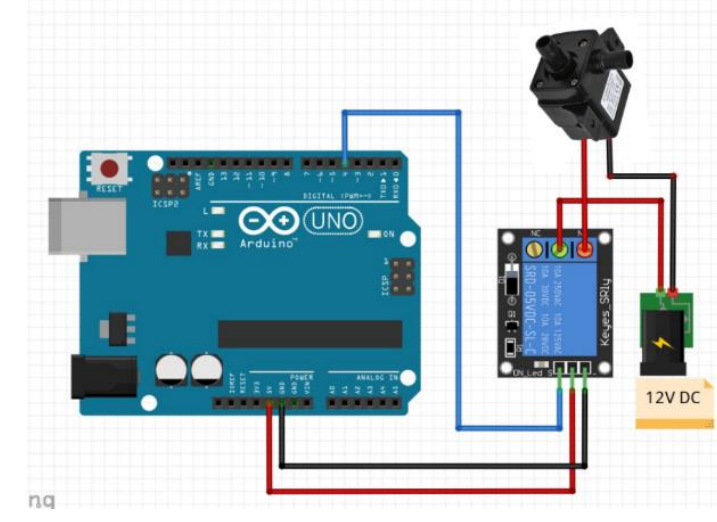

Fig. 7. Wiring diagram of the water pump with the Arduino

\subsection{Monitoring screen}

The Nextion display is used to display the measured values of temperature, humidity and light intensity. As the screen is touch-sensitive, functions have been added to display the optimal temperature and humidity values according to which certain actuators are switched $\mathrm{ON}$ or OFF. For proper display operation, the $5 \mathrm{~V}$ pin is connected to $5 \mathrm{~V}$ and the GND pin to the ground. Communication with the Arduino platform is achieved through a serial connection by connecting the TX pin to the RX and the RX pin to the TX pin of the Arduino platform. 


\section{System testing}

Figure 8 shows a schematic diagram of all the necessary parts connected into one whole. Each component must be connected according to the instructions in the figure and it is necessary to pay attention to the voltage levels of a particular component. For better visibility, but also easier connection of additional components such as resistors, DC power supply $12 \mathrm{~V}$ and MOSFET transistor, a prototype board was used on which the components are soldered.

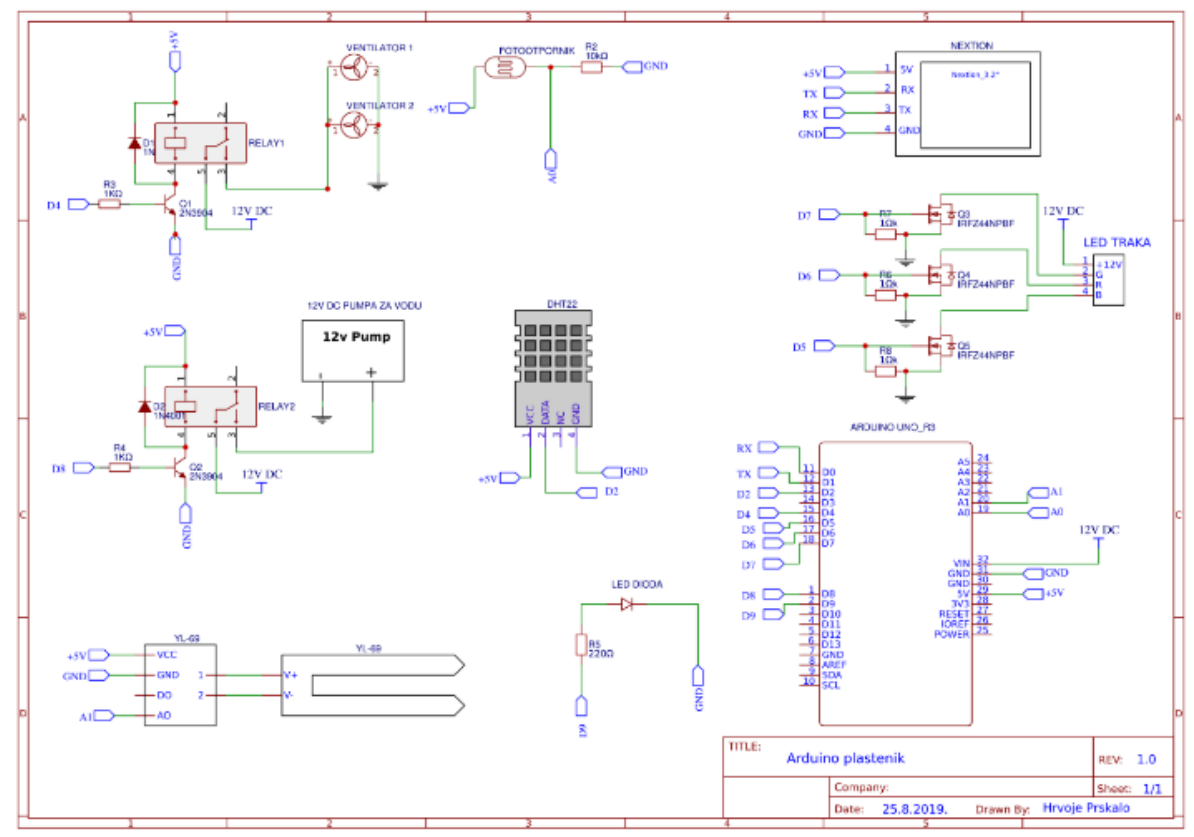

Fig. 8. Schematic representation of the finished system

Figure 9 shows a prototype plate housed in a plastic box. Figure 10 shows the finished layout of the greenhouse automation system with all the necessary parts. The model is made of acrylic glass on a wooden stand. The pump (whose motor is brushless) is connected to the water tank on one side via a hose, and the other end leads to the ground inside the greenhouse model. The fans are placed so that one blows in and the other blows out for the best possible air circulation. LED lighting is placed on top of the greenhouse model. The photo resistor is placed on the top outside the model so that it is not in the shadows. The temperature and relative humidity sensor and the soil moisture sensor are placed inside the greenhouse model. [8]

The screen receives the measured data every second via serial communication with the Arduino platform and displays them on certain parts of the screen itself. Depending on the crop of the plant, the optimum values of temperature and humidity are set over the display according to which the executive members are switched ON or OFF. According to the measured values, the current temperature is $30.5^{\circ} \mathrm{C}$ and exceeds the maximum set optimum temperature, so the ventilation and cooling fans are switched on. Relative humidity is shown in the value from 1 to $100 \%$ and is shown to be $57.2 \%$. Soil moisture is also displayed in a percentage of 1 to 100 and is $61 \%$, which means that it is not necessary to irrigate the soil and the pump is turned off. As in the previous two cases, the measured values of light intensity are scaled from 1 to 100 and it is seen that the measured value is 94 which means that the lighting is off. The test result can be seen in Figure 11.

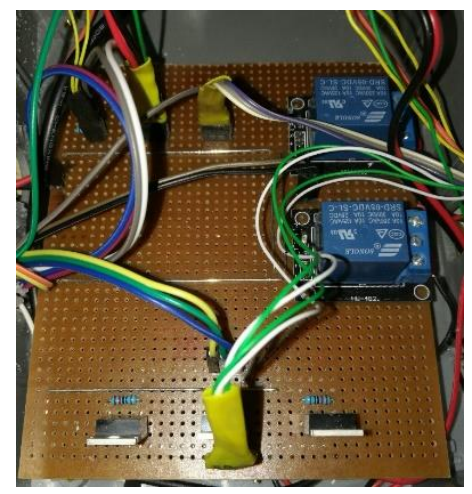

Fig. 9. Experimental tile with parts 


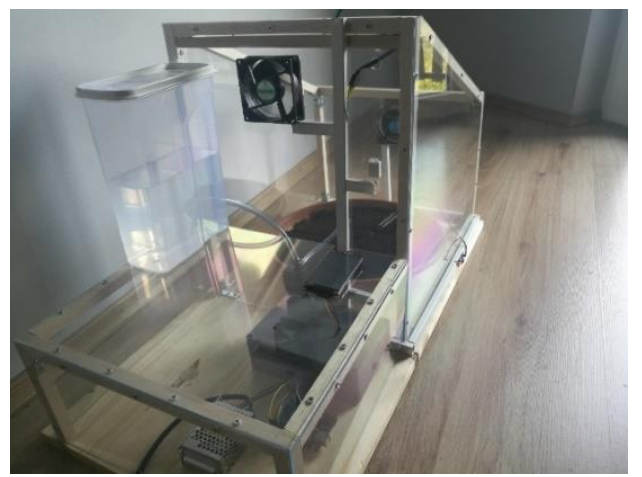

Fig. 10. Appearance of the finished system model

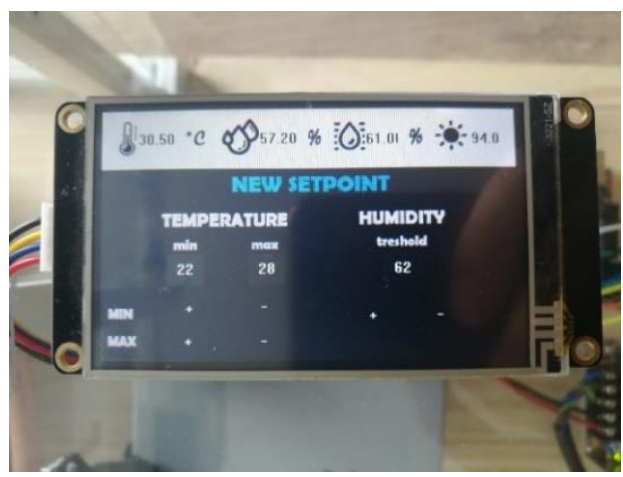

Fig. 11. Appearance of the screen with measured values

\section{Conclusion}

Crop production in sheltered areas has proven to be more efficient than outdoor production. In order to increase the yields and the quality of the production itself, systems for automatic control of microclimatic conditions inside the greenhouse are being introduced. So the idea is that under controlled conditions the yields increase. First, in a conversation with fellow biologists, it was defined which parameters we can influence in order to increase the yield: temperature, humidity and light. With this in mind, the appropriate sensors were selected. One of such systems is realized in this paper and is based on the Arduino platform which is acceptable for the price and its capabilities. As different plant cultures need different microclimatic conditions, a touch screen has been implemented in the system through which the optimal limits of microclimatic conditions can be easily changed. As the Arduino system is used, it is later possible to upgrade with different sensors depending on the needs. Testing of the system showed that the selected sensors proved to be satisfactory, that is, according to their characteristics, they satisfied the need for measurement within the previously planned limits. The accuracy of the sensors themselves is determined by calibrating them. This system is not limited in terms of use to specific crops due to its flexibility to change parameters. This emphasizes its universality.In the next step, the system will be implemented and monitor changes in yields compared to the conventional way of growing crops. Plant growth will be monitored on a monthly basis and compared to plant growth in uncontrolled conditions.

\section{References}

[1] Plastenik/Greenhouse, http://www.bilje.hr/POLJOPRIVREDA/AgBase_3/HTM/plastenik.html (14.8.2019.)

[2] Džijan P. (2017.), Web sustav za upravljanje mikroklimom plastenika na temelju podatkovne fuzije/ Web system for greenhouse microclimate management based on data fusion https://repozitorij.etfos.hr/islandora/object/etfos:1458/preview (14.8.2019.)

[3] Upravljanje mikroklimatskim uvjetima u zaštićenom prostoru/ Management of microclimatic conditions in a protected area, https://cdn.agroklub.com/upload/documents/upravljanje-mikroklimatskim-uvjetima.pdf (16.8.2019.)

[4] Transpiracija/ Transpiration, https://www.kako.hr/b/kako-se-odvija-transpiracija (12.9.2019.)

[5] Malčić G. (2012.), Sustavi nadzora i upravljanja industrijskih postrojenja/ Monitoring and control systems for industrial plants http://seminar.tvz.hr/materijali/materijali13/13E07.pdf (13.8.2019.)

[6] Arduino tutorials, https://www.arduino.cc/en/Tutorial/HomePage?from=Main.Tutorials (10.8.2019.)

[7] Getting Started with Arduino products, https://www.arduino.cc/en/Guide/HomePage (10.8.2019.)

[8] Pametni plastenik baziran na Arduino platformi/ Smart greenhouse based on Arduino platforms https://repozitorij.mev.hr/en/islandora/object/mev:1094

[9] https://www.savjetodavna.hr/wp-content/uploads/publikacije/investicijeupoljoprivredi.pdf (20.08.2020.)

[10] Prof. D.O.Shirsath, Punam Kamble, Rohini Mane, Ashwini Kolap, Prof.R.S.More, IOT Based Smart Greenhouse Automation Using Arduino, International Journal of Innovative Research in Computer Science \& Technology (IJIRCST) ISSN: 2347-5552, Volume-5, Issue-2, March 2017 DOI: 10.21276/ijircst.2017.5.2.4 